POS $\quad$ PROCEEDINGS

\title{
Gamma-Ray Beacons at the Dawn of the Universe
}

\section{Dario Gasparrini*}

Space Science Data Center - Agenzia Spaziale Italiana, I-00133 Roma, Italy

Istituto Nazionale di Fisica Nucleare, Sezione di Perugia, I-06123 Perugia, Italy

E-mail: dario.gasparriniessdc.asi.it

\section{Vaidehi S. Paliya and Marco Ajello}

Department of Physics and Astronomy, Clemson University, Kinard Lab of Physics, Clemson, SC 29634-0978, USA

E-mail: vpaliya@g.clemson.edu, majello@g.clemson.edu

\section{Roopesh Ojha}

NASA Goddard Space Flight Center, Greenbelt, MD 20771, USA

E-mail: roopesh.ojha@gmail.com

\section{on behalf of the Fermi-LAT Collaboration}

Gamma ray detected high-redshift blazars $(z>3)$ are intrinsically interesting since they inform us about the evolution of gamma-ray blazars and are, by definition, some of the more luminous blazars in the Universe. It has been found in many studies that such high z blazars host extremely massive black holes $\left(M_{\mathrm{BH}}>10^{9} M_{\odot}\right)$ and thus shed a new light on the formation of supermassive black holes in the early Universe. Here we report the first detection of gamma-ray emitting blazars beyond $\mathrm{z}=3.1$ using the sensitive Pass 8 dataset of Fermi-LAT. These objects are found to host extremely massive black holes at their centers, as confirmed from both IR-UV continuum modelling with a standard accretion disk and also with the emission line measurements using optical spectroscopy. Further details of the results will be presented within the framework of the disk-jet connection in powerful jetted AGNs.

35th International Cosmic Ray Conference - ICRC2017

10-20 July, 2017

Bexco, Busan, Korea

*Speaker. 


\section{Introduction}

Blazars are the most powerful Active Galactic Nuclei (AGN) with relativistic jets oriented close to the line of sight. The jet radiation dominates their broadband emission, especially at $\gamma$ rays where the inverse Compton (IC) hump of the blazar spectral energy distribution (SED) peaks around tens or hundreds of MeV. The Large Area Telescope (LAT) onboard the Fermi satellite [1] has already detected thousands of blazars, thus confirming that they are the most numerous population in the $\gamma$-ray sky [2]. Nonetheless, high-redshift blazars above a redshift of 3.1 are missing in the Fermi catalogs, possibly due to the shift of the IC peak to lower frequencies in which LAT is less sensitive. The newly released Pass 8 photon data set [3], with an improved eventlevel analysis, substantially enhances the sensitivity of the LAT at all energies and in particular at lower energies (i.e., $<200 \mathrm{MeV}$ ). This increases the capability of the LAT to detect spectrally soft, potentially high- $z$ blazars.

These objects typically have large bolometric luminosities $\left(L_{\text {bol. }}>10^{48} \mathrm{erg} \mathrm{s}^{-1}\right)$ and host powerful relativistic jets $\left[P_{j} \gtrsim \dot{M} c^{2}\right.$ for a given accretion efficiency; e.g., 4]. In general, they harbor extremely massive black holes $\left[M_{\mathrm{BH}} \sim 10^{9} \mathrm{M}_{\odot} ; 5\right]$. Since blazars are highly beamed, the detection of a single blazar implies the existence of $2 \Gamma^{2}$ (i.e., $\sim 400-600, \Gamma$ is the bulk Lorentz factor) misaligned blazars with similar properties. Therefore, the detection of high- $z$ blazars can constrain models of supermassive black hole formation in the early Universe [see, e.g., 6]. This suggests that the detection of new high- $z$ blazars will test the hypotheses of blazar evolution, since these high redshifts constrain the time available for such extreme objects to grow.

\section{Sample selection and Analysis}

In order to search for high- $z$ blazars, we start from the $\sim 1.4$ million quasars included in the Million Quasar Catalog [7]. We select all $z>3.1$ sources and retain only radio-loud (RL) quasars with $R>10$, where $R$ is the ratio of the rest-frame $5 \mathrm{GHz}$ to optical $B$ band flux density These 1103 objects represent our parent sample and we analyze LAT data for all of them according to the following procedure.

For each object we use $\sim 92$ months (from 2008 August 5 to 2016 April 1) of Fermi Pass 8 source class photons and analyze them following the standard data reduction procedure ${ }^{1}$. The details about the region of interest (ROI) size, background models used can be found in [8]. We consider a source to be significantly detected if the maximum likelihood test statistic (TS) is greater than $25[4.2 \sigma ; 9]$.

Since we are dealing with faint sources, we modify the standard data reduction procedure as follows. We expand the energy range considered so it spans $60 \mathrm{MeV}$ to $300 \mathrm{GeV}$. This allows the analysis to be more sensitive to spectrally soft $\gamma$-ray sources, i.e., blazars whose high-energy peak is shifted to lower energies $(\sim 1-10 \mathrm{MeV})$ as typical for high- $z$ blazars. Moreover, Pass 8 provides an increase in the acceptance at $<100 \mathrm{MeV}$ by up to $75 \%$, with respect to Pass 7 , which translates into an improved sensitivity for high- $z$ blazars. Because the energy resolution becomes increasingly worse at low energies we enable the energy dispersion correction in the analysis for all sources except the empirical diffuse models. A novelty introduced by Pass 8 is the characterization

\footnotetext{
${ }^{1}$ http://fermi.gsfc.nasa.gov/ssc/data/analysis/documentation/
} 
of the photons in PSF (point-spread function) type events, which sub-classify the events into four quartiles by quality of angular reconstruction, with the lowest quartile (PSF0) and highest quartile (PSF3) having the worst and the best, respectively, direction reconstruction. In order to take full advantage of this improvement, we perform a component-wise data analysis for each PSF event type by considering the product of the likelihood function (sum of the logarithms) for the four PSF event types, using the SUMMED likelihood method of the Science Tools ${ }^{2}$.

As in the case of our target sources, there could be faint gamma-ray emitters present in the data but not in the 3FGL catalog [10]. Thus, we rely on an iterative procedure to discover, localize, and insert such sources into the sky model. This is done by generating a residual TS map for each ROI. The spatial positions of unmodeled excesses with $\mathrm{TS} \geq 25$ are optimized and inserted into the sky model with power-law spectra. This procedure is iterated until no significant unmodeled emission remains in the ROI.

\section{Results}

Our systematic search for significant $\gamma$-ray emitters among a large sample of RL quasars has led to the detection of five sources. A likelihood ratio (LR) method [see 11, for details] associates the detected $\gamma$-ray sources and their radio counterparts [from NRAO VLA Sky Survey or NVSS; 12] with high confidence (association probability $>80 \%$ ). By repeating the LAT analysis adopting 1000 random positions drawn from a randomized NVSS catalog, we found that the probability that any of the newly detected $\gamma$-ray sources are spurious is negligible. The basic information for these objects is presented in Table 1 where we also show the results of the LAT data analysis. As can be seen, all the objects are extremely RL and $\gamma$-ray luminous quasars. According to our analysis, NVSS J151002+570243 $(z=4.31)$ is now the farthest known $\gamma$-ray emitting blazar.

In general, high-redshift blazars are brighter at hard X-rays than in the $\gamma$-ray band [e.g., 13], probably due to the shift of the blazar SED to lower frequencies. This could be due to the intrinsic shift of the high-energy peak to lower energies as the bolometric non-thermal luminosity increases [14]. Another possible reason for the shift could be the fact that the high-energy emission of such high-redshift blazars is powered via IC scattering off photons from the torus rather than the broadline region (BLR), which also contributes to the lowering of the frequency of their SED peak [15]. Alternatively, the SED peaks can also shift to lower energies provided the emission region is within the dense BLR photon field. In this case, an efficient cooling of the emitting electrons will cause the lowering of the SED peaks [16]. The shift of the SED causes their $\gamma$-ray spectra to become steeper and to move slightly outside, or at the limit, of the Fermi-LAT band. Indeed, all the blazars discovered here exhibit steep $\gamma$-ray spectra $\left(\Gamma_{\gamma}>2.5\right.$, see Table 1). This suggests their IC peak lie at $\mathrm{MeV}$ energies.

In Figure 1, we compare these newly detected distant objects with the blazars included in the third catalog of Fermi-LAT detected AGN [3LAC; 2]. As can be seen in the left panel, these sources occupy the region of high $\gamma$-ray luminosities $\left(L_{\gamma}>10^{47} \mathrm{erg} \mathrm{s}^{-1}\right)$ and soft photon indices $\left(\Gamma_{\gamma}>2.5\right)$, typical of powerful blazars. The right panel of Figure 1 compares the redshift distributions of these newly discovered high- $z$ blazars with that of the 3LAC blazars. Though the population of distant

\footnotetext{
${ }^{2} \mathrm{http} / / /$ fermi.gsfc.nasa.gov/ssc/data/analysis/software/
} 
blazars is small, this work opens a window for the study of high- $z$ blazars and may have a major impact on constraining the various physical parameters associated with the blazar population [see, e.g., 17, for a relevant discussion].

In order to understand the broadband behavior of these high- $z$ blazars we look into the literature for multi-frequency information. Though there is a paucity of such data, we found a few noteworthy observations that support the blazar nature of these objects. NVSS J064632+445116 was predicted as a candidate $\gamma$-ray emitter by [18], whereas NVSS J135406-020603 is included in the ROMA-BZCAT [19]. The quasar NVSS J212912-153841 is a hard X-ray spectrum luminous blazar and included in the 70 month Swift-Burst Alert Telescope catalog [20]. NVSS $\mathrm{J} 151002+570243$ is one of the best studied among all of the objects and exhibits an intense and hard X-ray spectrum [e.g., 21].

We generate broadband SEDs of the three objects that have archival X-ray observations and model them using a simple one zone synchrotron-IC emission approach prescribed in [22]. More details on the modelling and the different contributions to the IC peak can be found in [8]. The calculated jet powers and SED parameters are given in Table 2 and the modeled SEDs are shown in Figure 2.

In each of the three objects, the IR-UV emission is found to be dominated by an extremely luminous accretion disk $\left(L_{\text {disk }}>10^{46} \mathrm{erg} \mathrm{s}^{-1}\right)$. The X-ray spectra, on the other hand, are hard and the entire X-ray to $\gamma$-ray band of the SED can be explained by the IC scattering off the photons originating from the BLR. A strong accretion disk radiation implies a dense BLR photon field surrounding the jet, which in turn is observable in the form of broad optical emission lines. According to our SED modeling analysis, a large BLR radiative energy density indicates that most of the high-energy emission originates from the interaction of the BLR photons with the jet electrons. This suggests that the cooling of the electrons will be efficient, and accordingly the synchrotron emission will peak at low frequencies, which is supported by the modeling results. This indicates the location of the $\gamma$-ray emitting region to be inside the BLR. However, it should be noted that with the sparse available observations, it is not possible to tightly constrain the location of the emission region. The Compton dominance (ratio of the IC to synchrotron peak luminosities) of each of the three sources is also very large ( $>20$, Table 2$)$, a characteristic feature exhibited by powerful blazars. Other SED parameters are similar to those generally observed in high- $z$ blazars [e.g., 5]. Though the data used here are mostly non-simultaneous, they indicate a typical state of the blazar rather than any period of specific activity. Also, the $\gamma$-ray photon statistics are not good enough to search for temporal variability. Overall, the Fermi-LAT detection and the available data confirm the blazar nature of the 5 high $-z$ RL quasars.

\section{Discussion}

Powerful blazars are generally found to host massive black holes at their centers. It is, therefore, of great interest to determine the black hole mass of these $\gamma$-ray detected quasars. In Table 1 , we report the black hole masses for each of the five sources using information that we could find/derive from the optical spectroscopic information available in the literature [23]. Furthermore, we also derive the masses by modeling the observed IR-UV emission for three objects (Figure 2) with a standard [24] accretion disk and the results are presented in Table 2. Both methods predict 
the existence of massive black holes $\left(\sim 10^{8-10} \mathrm{M}_{\odot}\right)$ and match reasonably well within a factor of $\sim$ three $^{3}$, except for NVSS J151002+570243, for which the modeling approach predicts a higher black hole mass. In particular, the object NVSS J212912-153841 hosts one of the most massive black holes, $\sim 7 \times 10^{9} \mathrm{M}_{\odot}$, ever found in $\gamma$-ray emitting blazars, confirmed both from optical spectroscopic and disk modeling approaches.

At redshifts between 3 and 4 , the space density of black holes with $M_{\mathrm{BH}}>10^{9} \mathrm{M}_{\odot}$ hosted in jetted AGN is $50 \mathrm{Gpc}^{-3}$ [26]. This estimate is based on the luminosity function reported in [27], which, at those redshifts, relies only on five blazars. This work finds two more blazars hosting massive black holes $\left(M_{\mathrm{BH}}>10^{9} \mathrm{M}_{\odot}\right)$ in the same redshift range, considering black hole masses derived from the optical spectroscopic information. Adopting $\Gamma=13$, derived from our SED modeling, these two objects imply the presence of $\sim 675$ (i.e., $2 \times 2 \Gamma^{2}$ ) similar systems, but with jets pointing in all directions, in the same redshift bin. This brings the estimate of the space density of massive black holes hosted in jetted systems to $70_{-25}^{+37} \mathrm{Gpc}^{-3}$. Already at redshift 4 , this implies that there is a similar number of massive black holes hosted in radio-loud and radio-quiet systems and that, given their strong evolution, above that redshift most massive black holes might be hosted in radio-loud systems [28]. This clearly shows that the radio-loud phase may be a key ingredient for quick black hole growth in the early Universe. To this end, the detection of high- $z$ blazars becomes very important. Currently, the most promising approaches are, (1) lowering the energy threshold of the LAT, and (2) using NUSTAR. However, the optimal instrument would be a sensitive all-sky $\mathrm{MeV}$ telescope, e.g., e-ASTROGAM [29] and AMEGO ${ }^{4}$.
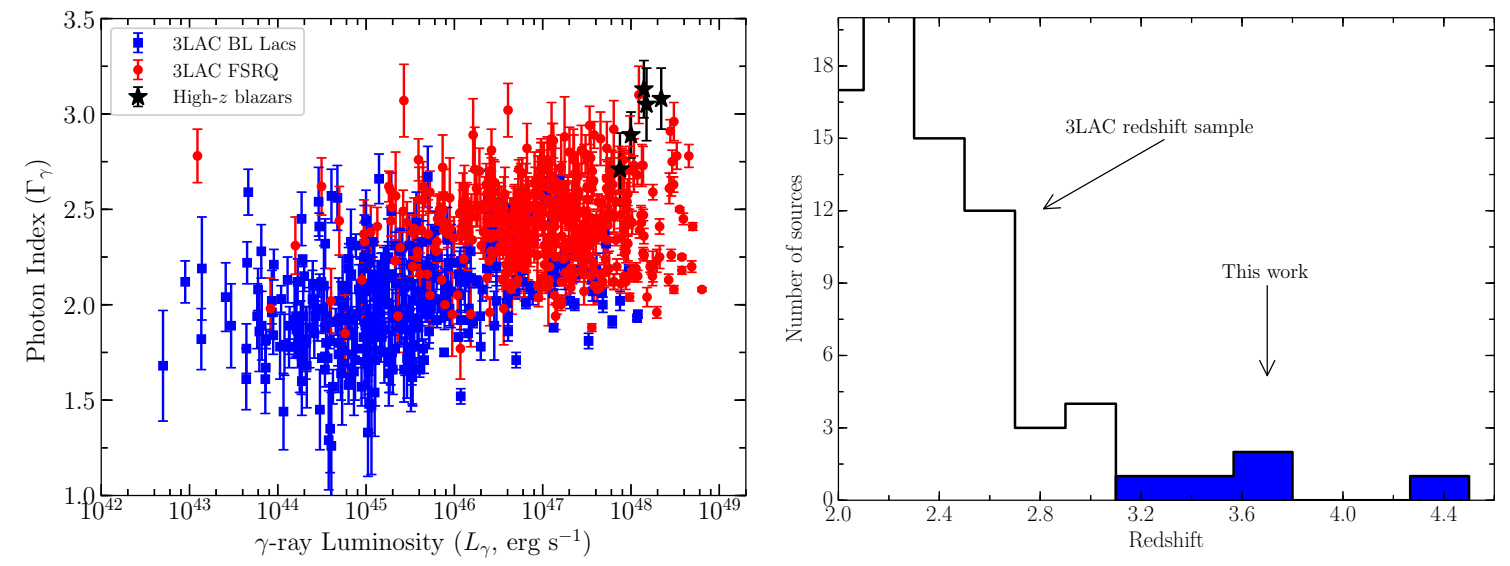

Figure 1 Comparison of new $\gamma$-ray detected high- $z$ blazars with 3LAC objects in, left: $\gamma$-ray luminosity vs. photon index plane, and right: the redshift histogram. The plotted $L_{\gamma}$ and $\Gamma_{\gamma}$ are derived for the $0.1-300 \mathrm{GeV}$ energy band, both for $3 \mathrm{LAC}$ and high- $z$ blazars newly detected in $\gamma$-rays, for an equal comparison.

\footnotetext{
${ }^{3}$ One should keep in mind the large uncertainties $(\sim 0.4 \mathrm{dex})$ associated with the virial BH mass estimation methods [see, e.g., 25].

${ }^{4}$ https://pcos.gsfc.nasa.gov/physpag/probe/AMEGO_probe.pdf
} 

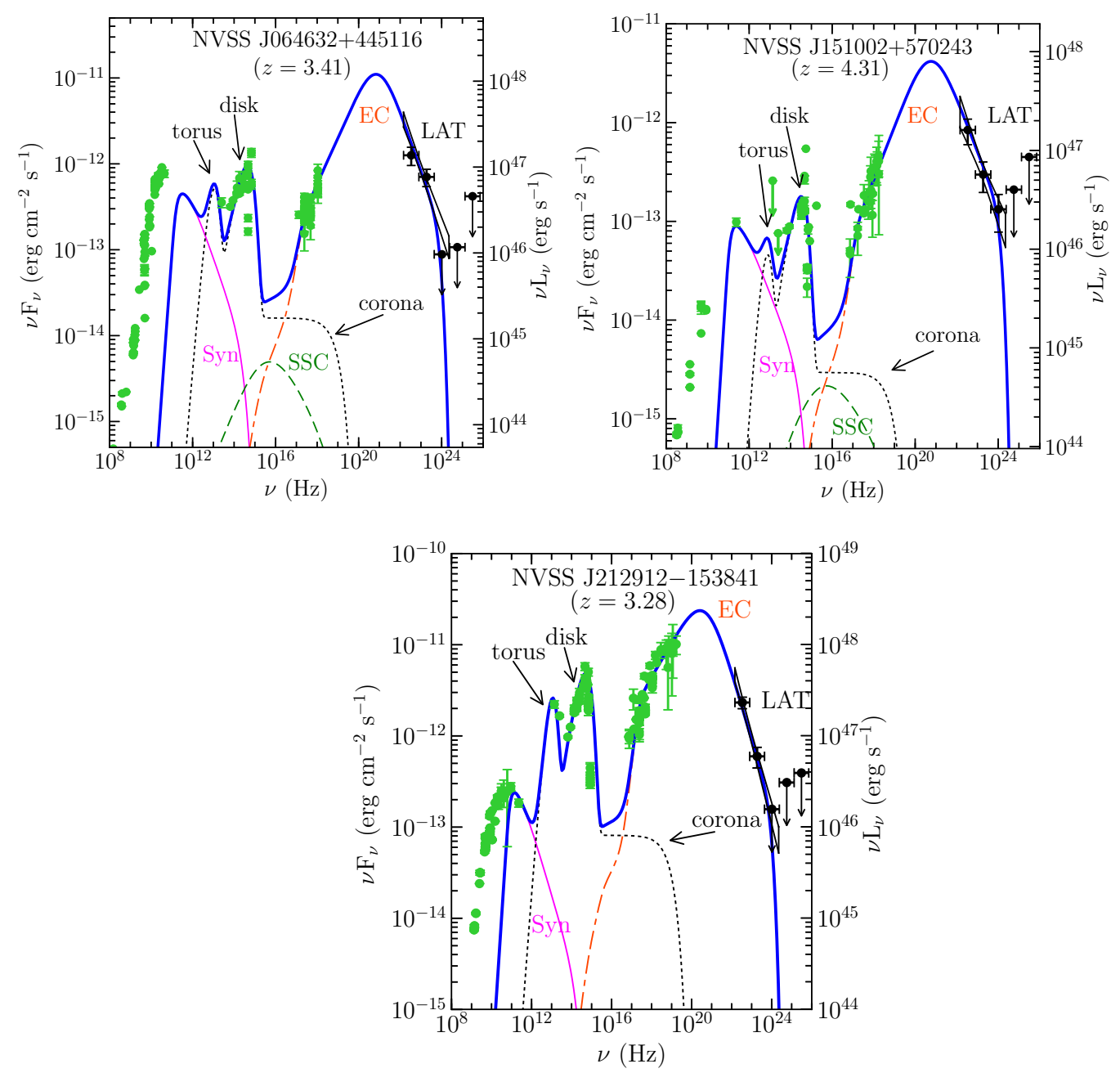

Figure 2 The broadband SEDs of three quasars reproduced using the one zone leptonic emission model. Lime green data points are the archival observations (http://tools.asdc.asi.it/SED/) and Fermi-LAT data points and bow-tie plots are in black. The dotted black line represents thermal radiations from the IR-torus, the accretion disk, and the X-ray corona, whereas, pink thin solid, green long dashed, and orange long-dash-dash-dot lines correspond to non-thermal synchrotron, SSC, and EC emissions, respectively. The blue thick solid line denotes the sum of the contributions from all the radiative components.

\section{Acknowledgments}

The Fermi-LAT Collaboration acknowledges support for LAT development, operation and data analysis from NASA and DOE (United States), CEA/Irfu and IN2P3/CNRS (France), ASI and INFN (Italy), MEXT, KEK, and JAXA (Japan), and the K.A. Wallenberg Foundation, the Swedish Research Council and the National Space Board (Sweden). Science analysis support in the operations phase from INAF (Italy) and CNES (France) is also gratefully acknowledged. This work performed in part under DOE Contract DE-AC02-76SF00515. Part of this work is based on archival data, software, or online services provided by the ASI Science Data Center (ASDC). 
Table 1. Basic information and results of the Fermi-LAT data analysis of high-redshift blazars.

\begin{tabular}{|c|c|c|c|c|c|c|c|c|c|}
\hline \multirow{3}{*}{$\begin{array}{l}\text { Name } \\
\text { (NVSS) }\end{array}$} & \multicolumn{3}{|c|}{ Basic information } & \multirow{3}{*}{ RL } & \multirow{3}{*}{$\begin{array}{c}M_{\mathrm{BH}, \mathrm{s}} \\
M_{\odot}\end{array}$} & \multicolumn{2}{|l|}{ Fermi-LAT data analysis } & \multirow[b]{2}{*}{$L_{\gamma}$} & \multirow{3}{*}{ TS } \\
\hline & Radio & n (J2000) & $z$ & & & $F_{0.06-300 \mathrm{GeV}}$ & $\Gamma_{\gamma}$ & & \\
\hline & hh mm ss.ss & dd mm ss.s & & & & $\left(10^{-8} \mathrm{ph} \mathrm{cm}^{-2} \mathrm{~s}^{-1}\right)$ & & $\left(10^{48} \mathrm{erg} \mathrm{s}^{-1}\right)$ & \\
\hline J064632+445116 & 064632.00 & +445117.0 & 3.4 & 1253 & $9.1^{\$}$ & $2.03 \pm 0.40$ & $2.68 \pm 0.10$ & $1.4 \pm 0.4$ & 62 \\
\hline J135406-020603 & 135406.90 & -020603.2 & 3.7 & 3741 & $8.9^{\dagger}$ & $2.32 \pm 0.52$ & $2.88 \pm 0.14$ & $2.5 \pm 0.8$ & 44 \\
\hline $\mathrm{J} 151002+570243$ & 151002.92 & +570243.4 & 4.3 & 1850 & $8.5^{\dagger}$ & $0.86 \pm 0.30$ & $2.55 \pm 0.16$ & $1.1 \pm 0.5$ & 34 \\
\hline $\mathrm{J} 163547+362930$ & 163547.24 & +362930.0 & 3.6 & 2842 & $8.7^{\dagger}$ & $5.31 \pm 0.96$ & $3.15 \pm 0.14$ & $7.0 \pm 0.2$ & 151 \\
\hline $\mathrm{J} 212912-153841$ & 212912.13 & -153841.0 & 3.3 & 263 & $9.8^{\$}$ & $2.65 \pm 0.43$ & $2.82 \pm 0.10$ & $1.9 \pm 0.4$ & 66 \\
\hline
\end{tabular}

Note. - Name, radio positions have been adopted from the NVSS catalog. Redshifts are taken from MQC. Radio-loudness (RL) is the ratio of the rest-frame 5 $\mathrm{GHz}$ (extrapolated from $1.4 \mathrm{GHz}$ assuming a flat radio spectrum) to optical $B$ band flux density. $M_{\mathrm{BH}, \mathrm{s}}$ is the logarithmic central black hole mass, in units of solar mass, derived/taken from available optical spectroscopic information: ${ }^{\$}[30],{ }^{\dagger}[23] . R_{95 \%}$ is the $95 \%$ error radius derived from the analysis. The $\gamma$-ray flux and apparent luminosity are in the energy range of $0.06-300 \mathrm{GeV}$.

Table 2 Summary of the parameters used/derived from the modeling of the SED of the objects shown in Figure 2. The viewing angle is taken as $3^{\circ}$ for all of them.

\begin{tabular}{|c|c|c|c|}
\hline Parameter & J0646+4451 & $\mathrm{J} 1510+5702$ & $\mathrm{~J} 2129-1538$ \\
\hline Slope of the electron energy distribution before break energy $(p)$ & 1.8 & 1.8 & 2.2 \\
\hline Slope of the electron energy distribution after break energy $(q)$ & 4.4 & 4.1 & 4.5 \\
\hline Magnetic field in Gauss $(B)$ & 2.1 & 1.4 & 1.3 \\
\hline Particle energy density in erg $\mathrm{cm}^{-3}\left(U_{e}^{\prime}\right)$ & 0.009 & 0.029 & 0.002 \\
\hline Bulk Lorentz factor $(\Gamma)$ & 12 & 11 & 14 \\
\hline Minimum Lorentz factor $\left(\gamma_{\min }^{\prime}\right)$ & 1 & 1 & 1 \\
\hline Break Lorentz factor $\left(\gamma_{b}^{\prime}\right)$ & 72 & 82 & 51 \\
\hline Maximum Lorentz factor $\left(\gamma_{\max }^{\prime}\right)$ & $2 \mathrm{e} 3$ & $3 \mathrm{e} 3$ & $2 \mathrm{e} 3$ \\
\hline Size of the emission region in parsec $\left(R_{\mathrm{blob}}\right)$ & 0.025 & 0.017 & 0.059 \\
\hline Dissipation distance in parsec $\left(R_{\text {diss }}\right)$ & 0.25 & 0.17 & 0.59 \\
\hline Size of the BLR in parsec $\left(R_{\mathrm{BLR}}\right)$ & 0.37 & 0.22 & 0.79 \\
\hline Black hole mass in log scale, in units of solar mass $\left(M_{\mathrm{BH}, \mathrm{m}}\right)$ & 9.60 & 9.48 & 9.85 \\
\hline Accretion disk luminosity in log scale $\left(L_{\text {disk }}, \mathrm{erg} \mathrm{s}^{-1}\right)$ & 47.11 & 46.65 & 47.78 \\
\hline Accretion disk luminosity in Eddington units $\left(L_{\text {disk }} / L_{\text {Edd }}\right)$ & 0.26 & 0.12 & 0.68 \\
\hline Fraction of the disk luminosity reprocessed by the BLR $\left(f_{\mathrm{BLR}}\right)$ & 0.1 & 0.1 & 0.1 \\
\hline Fraction of the disk luminosity reprocessed by the IR-torus $\left(f_{\mathrm{IR}}\right)$ & 0.5 & 0.5 & 0.5 \\
\hline Compton dominance (CD) & 25 & 47 & 99 \\
\hline Jet power in electrons in $\log$ scale $\left(P_{\mathrm{e}}, \mathrm{erg} \mathrm{s}^{-1}\right)$ & 44.88 & 44.94 & 45.14 \\
\hline Jet power in magnetic field in $\log$ scale $\left(P_{\mathrm{B}}\right)$, erg s ${ }^{-1}$ & 46.15 & 45.38 & 46.58 \\
\hline Radiative jet power in log scale $\left(P_{\mathrm{r}}, \mathrm{erg} \mathrm{s}^{-1}\right)$ & 45.89 & 45.70 & 46.31 \\
\hline Jet power in protons in log scale $\left(P_{\mathrm{p}}, \mathrm{erg} \mathrm{s}^{-1}\right)$ & 47.38 & 47.45 & 47.89 \\
\hline
\end{tabular}

\section{References}

[1] W. B. Atwood et al., The Large Area Telescope on the Fermi Gamma-Ray Space Telescope Mission, ApJ 697 (June, 2009) 1071-1102, [arXiv:0902.1089].

[2] M. Ackermann et al., The Third Catalog of Active Galactic Nuclei Detected by the Fermi Large Area Telescope, ApJ 810 (Sept., 2015) 14, [arXiv: 1501.0605 ].

[3] W. Atwood et al., Pass 8: Toward the Full Realization of the Fermi-LAT Scientific Potential, ArXiv e-prints (Mar., 2013) [arXiv:1303.3514].

[4] G. Ghisellini et al., The power of relativistic jets is larger than the luminosity of their accretion disks, Nature $\mathbf{5 1 5}$ 
(Nov., 2014) 376-378, [arXiv: 1411.5368].

[5] G. Ghisellini et al., Chasing the heaviest black holes of jetted active galactic nuclei, MNRAS 405 (June, 2010) 387-400, [arXiv:0912.0001].

[6] G. Ghisellini et al., The role of relativistic jets in the heaviest and most active supermassive black holes at high redshift, MNRAS 432 (July, 2013) 2818-2823, [arXiv: 1304 . 1152].

[7] E. W. Flesch, The Half Million Quasars (HMQ) Catalogue, PASA 32 (Mar., 2015) e010, [arXiv: 1502 . 0630].

[8] M. Ackermann et al., Gamma-ray Blazars Within the First 2 Billion Years, ApJ 837 (Mar., 2017) L5, [arXiv:1702.0400].

[9] J. R. Mattox et al., The Likelihood Analysis of EGRET Data, ApJ 461 (Apr., 1996) 396.

[10] F. Acero, et al., Fermi Large Area Telescope Third Source Catalog, ApJS 218 (June, 2015) 23, [arXiv:1501.0200].

[11] M. Ackermann et al., The Second Catalog of Active Galactic Nuclei Detected by the Fermi Large Area Telescope, ApJ 743 (Dec., 2011) 171, [arXiv: 1108 .1420].

[12] J. J. Condon et al., The NRAO VLA Sky Survey, AJ 115 (May, 1998) 1693-1716.

[13] T. Sbarrato et al., NuSTAR Detection of the Blazar B2 1023+25 at Redshift 5.3, ApJ 777 (Nov., 2013) 147, [arXiv:1309.3280].

[14] G. Fossati et al., A unifying view of the spectral energy distributions of blazars, MNRAS 299 (Sept., 1998) 433-448, [astro-ph/9804103].

[15] M. Sikora et al., On the Nature of MeV Blazars, ApJ 577 (Sept., 2002) 78-84, [a stro-ph / 0205527 ].

[16] G. Ghisellini et al., A theoretical unifying scheme for gamma-ray bright blazars, MNRAS 301 (Dec., 1998) 451-468, [astro-ph/9807317].

[17] V. S. Paliya et al., Broadband Observations of High Redshift Blazars, ApJ 825 (July, 2016) 74, [arXiv:1604.0856].

[18] S. E. Healey et al., CGRaBS: An All-Sky Survey of Gamma-Ray Blazar Candidates, ApJS 175 (Mar., 2008) 97-104, [arXiv:0 709.1735$]$.

[19] E. Massaro et al., The 5th edition of the Roma-BZCAT. A short presentation, Ap\&SS 357 (May, 2015) 75, [arXiv:1502.0775].

[20] W. Baumgartner et al., The 70 Month Swift-BAT All-sky Hard X-Ray Survey, ApJS 207 (Aug., 2013) 19, [arXiv:1212.3336].

[21] J. Wu et al., An X-Ray and Multiwavelength Survey of Highly Radio-loud Quasars at $z>4$ : Jet-linked Emission in the Brightest Radio Beacons of the Early Universe, ApJ 763 (Feb., 2013) 109, [arXiv: 1301.0012].

[22] G. Ghisellini and F. Tavecchio, Canonical high-power blazars, MNRAS 397 (Aug., 2009) 985-1002, [arXiv:0902.0793].

[23] S. Alam et al., The Eleventh and Twelfth Data Releases of the Sloan Digital Sky Survey: Final Data from SDSS-III, ApJS 219 (July, 2015) 12, [arXiv: 1501 . 0096].

[24] N. I. Shakura and R. A. Sunyaev, Black holes in binary systems. Observational appearance., A\&A 24 (1973) 337-355.

[25] Y. Shen et al., A Catalog of Quasar Properties from Sloan Digital Sky Survey Data Release 7, ApJS 194 (June, 2011) 45, [arXiv:1006.5178].

[26] T. Sbarrato et al., Blazar candidates beyond redshift 4 observed by Swift, MNRAS 446 (Jan., 2015) 2483-2489, [arXiv:1410.0364].

[27] M. Ajello et al., The Evolution of Swift/BAT Blazars and the Origin of the MeV Background, ApJ 699 (July, 2009) 603-625, [arXiv:0905.0472].

[28] M. Volonteri et al., Blazars in the early Universe, MNRAS 416 (Sept., 2011) 216-224, [arXiv: 1103.5565$].$

[29] V. Tatischeff et al., The e-ASTROGAM gamma-ray space mission, in Space Telescopes and Instrumentation 2016: Ultraviolet to Gamma Ray, vol. 9905 of Proc. SPIE, p. 99052N, July, 2016. arXiv: 1608.0373.

[30] J. Torrealba et al., Optical Spectroscopic Atlas of the MOJAVE/2cm AGN Sample, Rev. Mexicana Astron. Astrofis. 48 (Apr., 2012) 9-40, [arXiv:1107.3416]. 